\title{
Fracture Nonunions and Delayed Unions Treated With Low-Intensity Pulsed Ultrasound Therapy: A Clinical Series
}

Kavyansh Bhan ${ }^{1}$, Ronak Patel ${ }^{1}$, Kamrul Hasan ${ }^{1}$, Mahesh Pimplé ${ }^{1}$, Sucheta Sharma ${ }^{2}$, Varsha Nandwana ${ }^{3}$ , Mhafrin Basta ${ }^{4}$

1. Trauma \& Orthopaedics, Barts Health NHS Trust, London, GBR 2. Orthopaedic Surgery, Punjab Institute of Medical Sciences, Jalandhar, IND 3. Orthopaedic Surgery, Lady Hardinge Medical College, Delhi, IND 4. Dermatology, James Cook University Hospital, Middlesbrough, GBR

Corresponding author: Kavyansh Bhan, kavyanshbhan1993@gmail.com

\begin{abstract}
The incidence of nonunion of fractures has been steadily rising owing to improved life expectancy following severe injuries along with rising cases of polytrauma. Once a nonunion is established, the chances of spontaneous healing are deemed to be quite low. Fracture nonunion continues to be a challenge in clinical practice with nonunions having a considerable impact on patient's quality of life causing both functional and psychosocial disability. Low-Intensity Pulsed Ultrasound (LIPUS) therapy is being projected as a viable and non-interventional alternative to surgical management of nonunions and delayed unions. LIPUS therapy is being widely recommended as a standalone treatment option for the treatment of established nonunions and delayed unions as it is believed to promote healing in all phases of fracture healing viz., inflammatory, intramembranous ossification, chondrogenesis, endochondral ossification and remodelling. In the current scenario of varying results and unclear clinical role of LIPUS therapy, we present a prospective case series of fracture nonunions and delayed unions treated with LIPUS therapy at a large District General Hospital.
\end{abstract}

Categories: Orthopedics, Trauma

Keywords: nonunions, delayed union, lipus therapy, ultrasound therapy, radiological and clinical fracture healing

\section{Introduction}

The incidence of nonunion of fractures varies between $5-10 \%$ and has been steadily rising owing to improved life expectancy following severe injuries [1]. The incidence of nonunion is also directly related to the severity of causative injury with multiply injured patients being at a higher risk of developing long-term orthopaedic complications such as nonunion [2-3]. Once a nonunion is established, the chances of spontaneous healing are deemed to be quite low [4]. Fracture nonunion and delayed unions continue to be a challenge in clinical practice with nonunions having a considerable impact on patient's quality of life causing functional and psychosocial disability along with it being the cause of an economic burden owing to prolonged disability and downtime of job [5]. Although ultrasound is routinely indicated as a diagnostic modality in Trauma \&

Review began 07/15/2021 Review ended 08/10/2021 Published 08/10/2021

\section{() Copyright 2021}

Bhan et al. This is an open access article distributed under the terms of the Creative Commons Attribution License CC-BY 4.0., which permits unrestricted use, distribution, and reproduction in any medium, provided the original author and source are credited.
Orthopaedics, its use as a therapeutic intervention is still not well understood [6].

The biological use of ultrasound was first explored by Wood and Lumis back in 1924 wherein they described the biological changes related to ultrasound treatment [7]. The first evidence of the use of ultrasound in the treatment of fracture nonunions however can be traced to 1983 wherein success rates of almost $70 \%$ were reported following the use of ultrasound therapy in established nonunions [8]. The high healing rates followed by a slew of successful clinical trials done in the United States (US) led to the approval of LowIntensity Pulsed Ultrasound (LIPUS) therapy by the US Food and Drug Administration in 1994 [9]. The approval was initially granted for accelerating the healing of certain fresh fractures but was later extended to encompass established nonunions in 2000 [9]. The approved ultrasound signal for LIPUS is the $1.5 \mathrm{MHz}$ sinusoidal wave modulated in bursts of $200 \mathrm{~ms}$ at a repetition frequency of $1 \mathrm{kHz}$. Recent literature has projected LIPUS as a safe and effective home treatment option for delayed unions and nonunions with success rates of up to 93\% [10]. LIPUS is being widely recommended as a standalone treatment option rather than an adjuvant for the treatment of established nonunions and delayed unions as it is believed to promote healing in all phases of fracture healing viz., inflammatory, intramembranous ossification, chondrogenesis, endochondral ossification, and remodelling [9]. Moreover, LIPUS is also being widely considered as a viable treatment option for nonunions and delayed unions in individuals who are not ideal candidates for surgery [11]. This includes patients who are suffering from dementia, old age, multiple organ failure, and coma [11]. In healthy individuals, however, Rutten et al. found that LIPUS was effective in reducing the mean time to the radiographic union by 39.8 days [12]. Zura et al. in their large observational cohort study of 767 patients reported a radiographic union rate of $80 \%$ in established nonunions where LIPUS was used as a standalone treatment modality without any adjuncts [13].

In the current scenario of varying results and unclear clinical role of LIPUS, we present a prospective case 
series of fracture nonunions and delayed unions treated with Low-Intensity Pulsed Ultrasound (LIPUS) therapy at a District General Hospital.

\section{Materials And Methods}

The LIPUS therapy has been in use for delayed unions and nonunions at our institute since 2016. A Trust pathway detailing indications and referral criteria is in place, and this was circulated amongst the members of the staff again.

\section{Inclusion criteria}

All patients having reached the age of 16 or above with established nonunions or delayed unions were included in the study. Patients who had not attained the age of 16 were not included as the role of LIPUS in nonunions or delayed unions of the immature skeleton is not well supported by the current body of literature [4]. A minimum period of six months post-surgery/post-injury was allowed for the fractures to heal, before being considered for LIPUS therapy. Only patients who were willing to accept LIPUS therapy as a standalone treatment option were included.

\section{Exclusion criteria}

Patients with unstable fractures or patients who were having a concomitant infection were excluded from the study. All patients with spinal fractures or skull fractures were also excluded. The patients who were not willing to accept the use of LIPUS therapy as a standalone treatment option were excluded. Patients in whom wound care or current wound status hindered ultrasound-skin contact were not included.

\section{Methods}

All prospective patients were seen face to face in the outpatient clinic for a clinical evaluation. Plain radiograph with standard Anteroposterior (AP) and Lateral views were obtained to establish the correct diagnosis. Nonunion was confirmed by the absence of any cortical or cancellous bone bridge between the fracture fragments. In instances where bone bridging was unclear on X-rays or there was a presence of bulky osteosynthesis material hindering appropriate diagnosis, Computed Tomography (CT) scan was obtained to establish the diagnosis. The patients were explained about the role of LIPUS, its proposed mechanism of action, and its principle. Once the patients agreed to the inclusion, they were provided with the portable LIPUS device, namely EXOGEN ${ }^{\circledR}$ (Bioventus LLC, London, United Kingdom) and treatment commenced. The patients were provided with training to correctly use the device at home. The training was delivered by the prescribing physician and a representative from the LIPUS device manufacturer's company. Leaflets with detailed instructions on how to use the device were also provided to the patients. Contact numbers of the relevant team members were given to the patients, in case of any queries.

The patients were explained that the treatment would require them to use the LIPUS device for 20 minutes every day. They were told that the device would need to be centred over the site of nonunion or delayed union. The target site for the LIPUS device was marked for reference on the affected site after an AP X-ray of the non-united fracture was taken with a radio-opaque mark on the skin corresponding to the fracture site. This was made on an easily accessible part of the affected site, for example, the anterior aspect of a limb. Visual Analog Scale (VAS) was also utilized to assess pain levels of the patients on commencement of the treatment and end of the treatment. The patients were followed up every three months for a minimum period of six months. Earlier appointments were also made at the request of patients. The maximum period for treatment with LIPUS was agreed to be one year. The patients were instructed to follow the LIPUS device instructions with at least $90 \%$ adherence to be deemed compliant with treatment.

A total of 49 patients were enrolled from December 2016 to June 2020 (42 months). The relationship between the radiographic union and LIPUS was analysed. The effect of fracture healing on the VAS score was also analysed. The case series was conducted with the aim to understand the efficacy of LIPUS therapy on fracture nonunions and delayed unions. It sought to assess the results of LIPUS therapy on nonunions and delayed unions along with the limitations of the therapy.

\section{Results}

The group of 49 patients enrolled between December 2016 to June 2020 comprised 16 males and 33 females aged from 24 years to 86 years (Table 1), with an average age of 54.61 years. All the enrolled patients were followed up for a minimum period of six months. All the patients had upper or lower extremity fractures with no patients with spinal or skull fractures recruited onto the study. The average time gap in the referral of patients from the date of injury to the first LIPUS therapy clinic appointment was 9.2 months (range 6-18 months).

\begin{tabular}{|l|l|l|}
\hline Age of Patient & Diagnosis & Exogen Duration \\
\hline 60 years & Nonunion Distal Radius & 6 months \\
\hline
\end{tabular}




\section{Cureus}

\begin{tabular}{|c|c|c|c|}
\hline 70 years & Delayed Union post Midshaft Humerus ORIF & 6 months & Union \\
\hline 70 years & Nonunion post Distal Femur Fracture ORIF & 12 months & Non-union \\
\hline 83 years & Delayed Union post Distal Radius ORIF & 12 months & Non-union \\
\hline 31 years & Delayed Union Tibial Shaft Fracture & 6 months & Union \\
\hline 45 years & Nonunion Tibial Shaft Fracture & 12 months & Union \\
\hline 59 years & Nonunion 5th Metatarsal Fracture & Lost to follow up & - \\
\hline 86 years & Nonunion post Midshaft Femur Fracture ORIF & 12 months & Non-union \\
\hline 50 years & Nonunion Post Distal Femur ORIF & 6 months & Union \\
\hline 53 years & Nonunion post 5th Metatarsal Fracture & 6 months & Union \\
\hline 31 years & Nonunion post Scaphoid Fracture & 6 months & Union \\
\hline 40 years & Delayed union post Distal Tibia Fracture & 6months & Union \\
\hline 70 years & Nonunion post Talus Fracture ORIF & 12 months & Non-union \\
\hline 42 years & Delayed union post 5th Metatarsal Fracture & 3 months & Union \\
\hline 60 years & Nonunion post Olecranon Fracture ORIF & 3 Months & Union \\
\hline 68 years & Nonunion Midshaft Humerus Fracture & 12 months & Non-union \\
\hline 44 years & Delayed union 5 th iMetatarsal $F$ racture & Lost to follow up & - \\
\hline 40 years & Delayed union post Tibial Shaft Fracture & 6 months & Union \\
\hline 51 years & Nonunion post 5 th metatarsal ORIF & 4 months & Union \\
\hline 56 years & Nonunion post 5 th Metatarsal fracture & 4 months & Union \\
\hline 54 years & Delayed union post Distal Radius Fracture ORIF & 3 months & Union \\
\hline 60 years & Nonunion post Ankle Weber B Fracture & 6 months & Union \\
\hline 45 years & Nonunion post Midshaft Femur Fracture ORIF & 6 months & Union \\
\hline 49 years & Nonunion post Midhsaft Femur Fracture ORIF & 6 months & Union \\
\hline 36 years & Delayed union 5th Metatarsal Fracture & 3 Months & Union \\
\hline 24 years & Delayed union 3rd Metatarsal + Cuneiform Fracture & 5 months & Union \\
\hline 63 years & Delayed union 5th Metatarsal fracture & 5months & Union \\
\hline 47 years & Nonunion post 1st Metatarsal ORIF & 6 months & Union \\
\hline 66 years & Delayed union Clavicle Fracture & 3 months & Union \\
\hline 55 years & Delayed union calcaneum Fracture & 3 months & Union \\
\hline 53 years & Nonunion post Midshaft Humerus Fracture ORIF & 12 months & Non-union \\
\hline 73 years & Nonunion post 5th Metatarsal Fracture & 6 months & Union \\
\hline 56 years & Nonunion post Ankle Weber B Fracture & 4 months & Union \\
\hline 77 years & Delayed union Post Distal Radius Fracture ORIF & 3 months & Union \\
\hline 26 years & Delayed union post Ankle Fracture ORIF (Weber B) & 5 months & Union \\
\hline 55 years & Delayed union 5th Metatarsal Fracture & 5 months & Union \\
\hline 54 years & Nonunion post Proximal Tibia Fracture ORIF & 12 months & Non-union \\
\hline 29 years & Nonunion post 5th Metatarsal Fracture ORIF & 3 months & Union \\
\hline 53 years & Nonunion post 1st Metatarsal Fracture ORIF & Lost to Follow up & - \\
\hline 43 years & Delayed union post $2 \mathrm{nd}, 3 \mathrm{rd}$, 4th Metatarsal Fracture & 6 months & Union \\
\hline
\end{tabular}




\section{Cureus}

\begin{tabular}{|llll|}
73 years & Delayed union Ankle Weber B Fracture & Lost to follow up & - \\
59 years & Nonunion post Distal Radius Fracture ORIF & 6 months & Union \\
76 years & Nonunion post Proximal Tibia Fracture ORIF & Noncompliant & - \\
61 years & Nonunion post Midshaft Humerus Fracture ORIF & Noncompliant & - \\
70 years & Nonunion post Talus Fracture ORIF & 12 months & Non-union \\
58 years & Nonunion post Ankle ORIF (Weber B) & 12 months & Non-Union \\
44 years & Delayed union post Midshaft Tibia Fracture ORIF & 5 months & Union \\
46 years & Nonunion post 1st Metatarsal Fracture ORIF & Noncompliant & - \\
62 years & Delayed union post Ankle Fracture ORIF (Weber B) & 6 months & Union \\
\hline
\end{tabular}

\section{TABLE 1: Summary of the patients recruited}

ORIF - Open Reduction and Internal Fixation; Exogen - Low-intensity pulsed ultrasound (LIPUS) device used for the therapy

Four patients were lost to follow up and three others were deemed to be noncompliant. The required threshold of $90 \%$ adherence was met by the remaining 42 patients. We noted mean compliance of $95 \%$ (range 91 - 97\%). In this cohort of 42 compliant patients, 18 patients were managed conservatively upon initial presentation to fracture clinic, while the remaining 24 patients underwent surgery as the first line of management.

Thirty-three patients out of 42 showed a radiographic union on X-rays at the end of LIPUS therapy treatment; for example, see Figures $1-6$. This is indicative of the $78.57 \%$ success rate of LIPUS in the treatment of nonunions. In the nine failed patients, there was no evidence of union despite being given the LIPUS treatment for the maximum pre-agreed period of one year. Out of these nine patients, one patient was post midshaft Humerus Open Reduction and Internal Fixation (ORIF), one patient post midshaft humerus fracture managed conservatively, one post Distal Femur ORIF, one post Distal Radius ORIF, one post midshaft Femur ORIF, one post Ankle ORIF, two post Talus ORIF and one post Proximal Tibia ORIF. 


\section{Cureus}

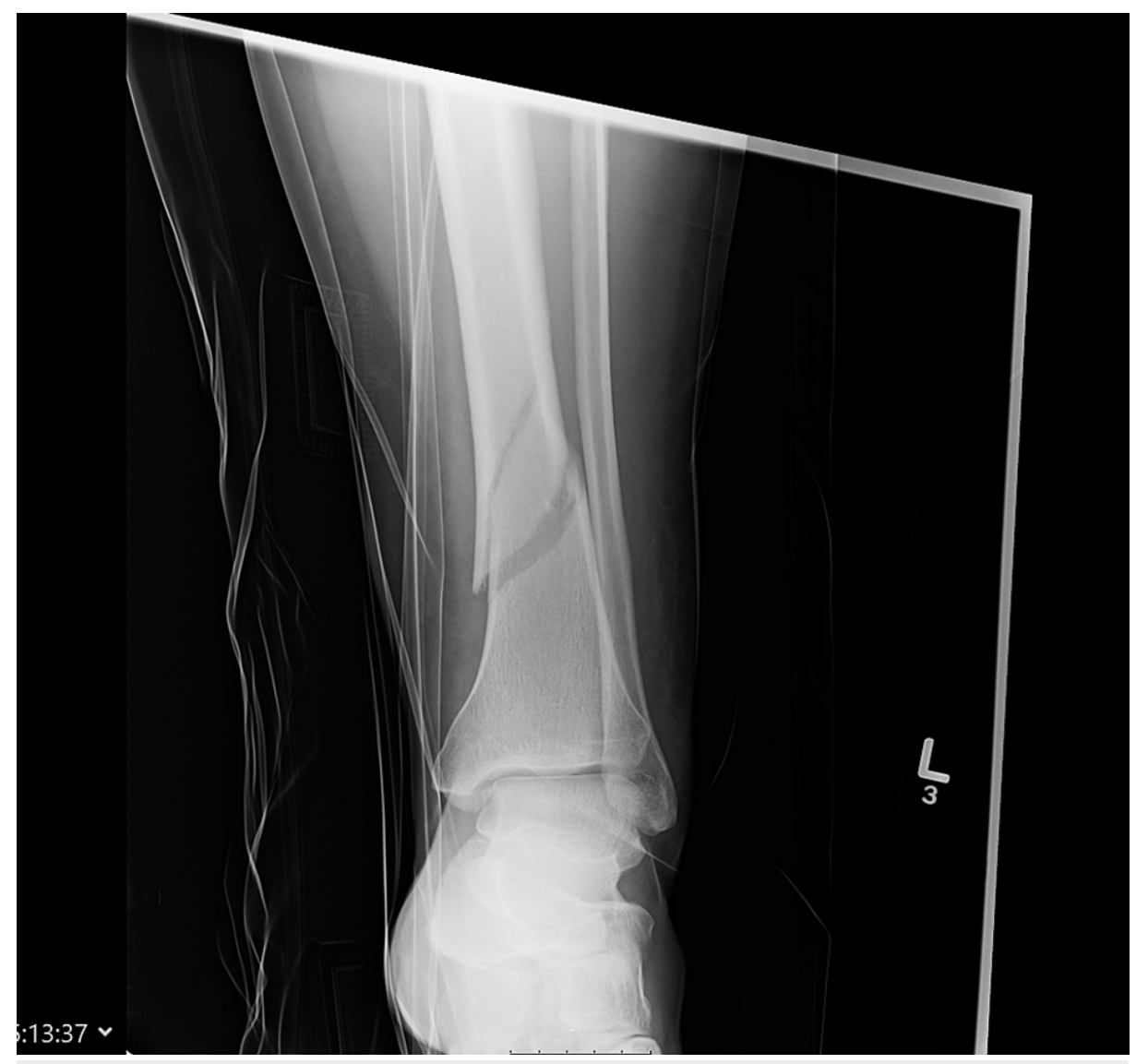

FIGURE 1: Distal Tibia fracture at the time of injury (AP View) AP - Anteroposterior 


\section{Cureus}

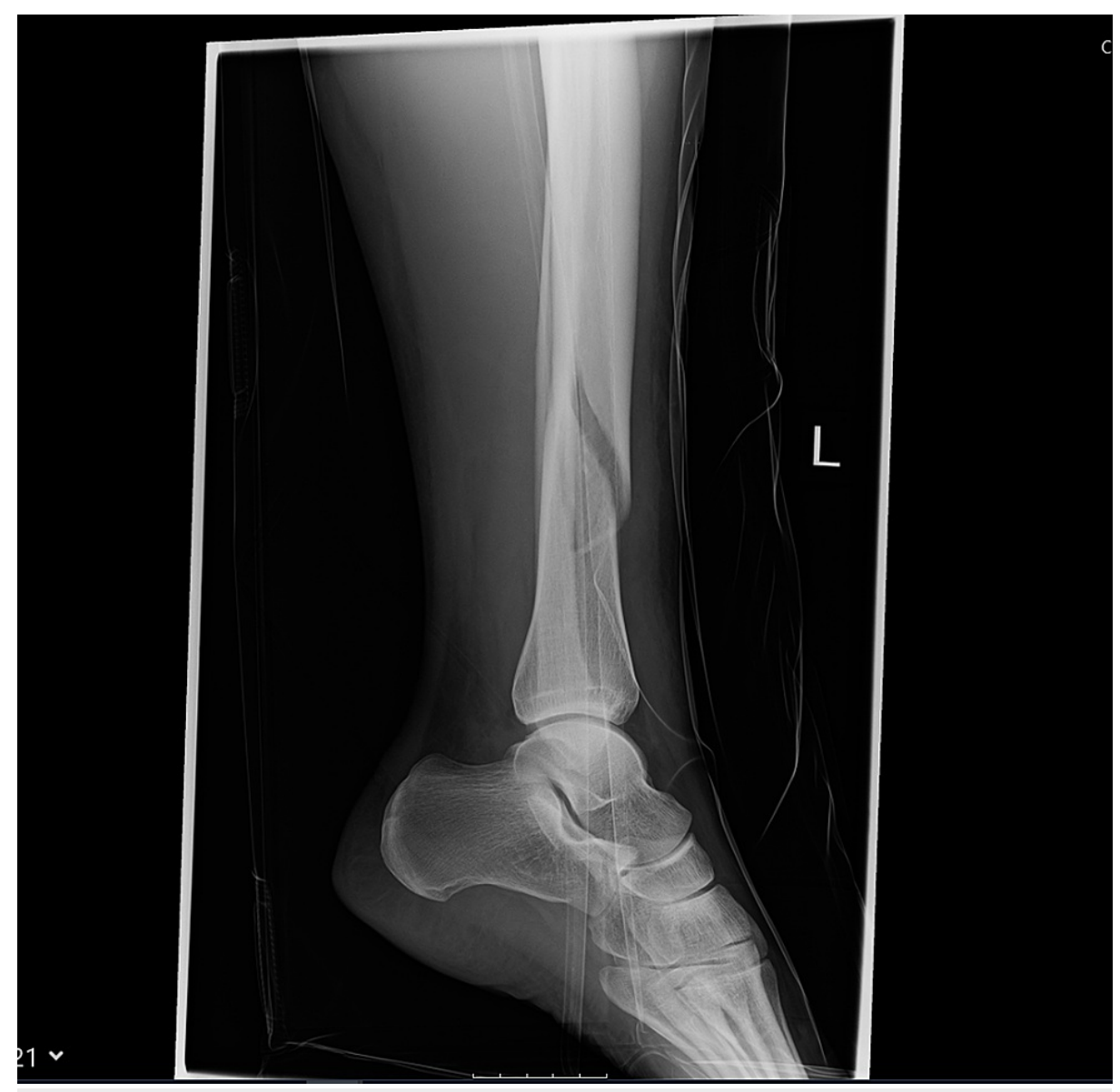

FIGURE 2: Distal Tibia fracture at the time of injury (Lateral view) 


\section{Cureus}

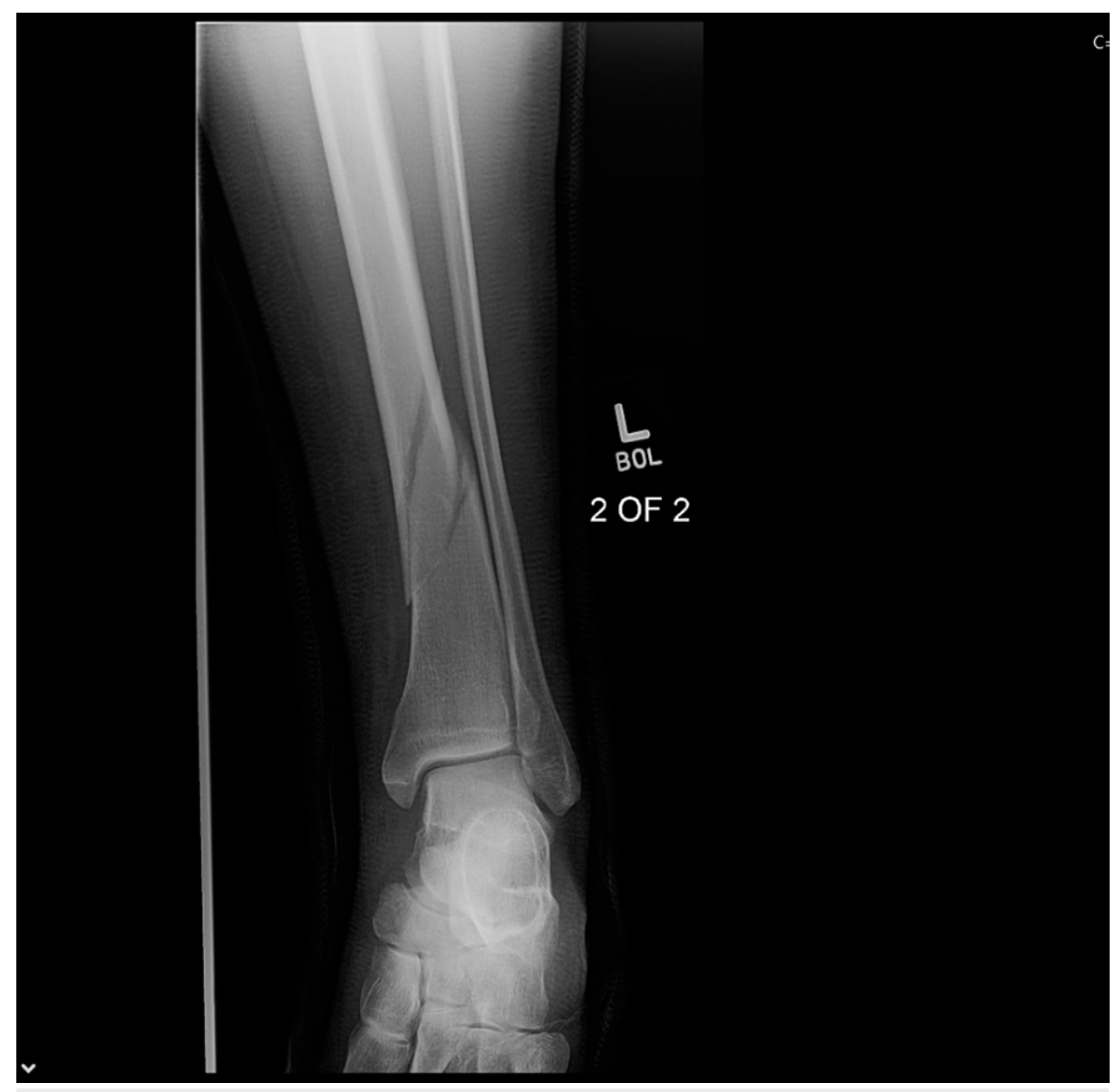

FIGURE 3: Distal Tibia fracture at the time of initiation of LIPUS therapy (6 months post injury - AP view)

AP - Anteroposterior; LIPUS - Low-Intensity Pulsed Ultrasound 


\section{Cureus}

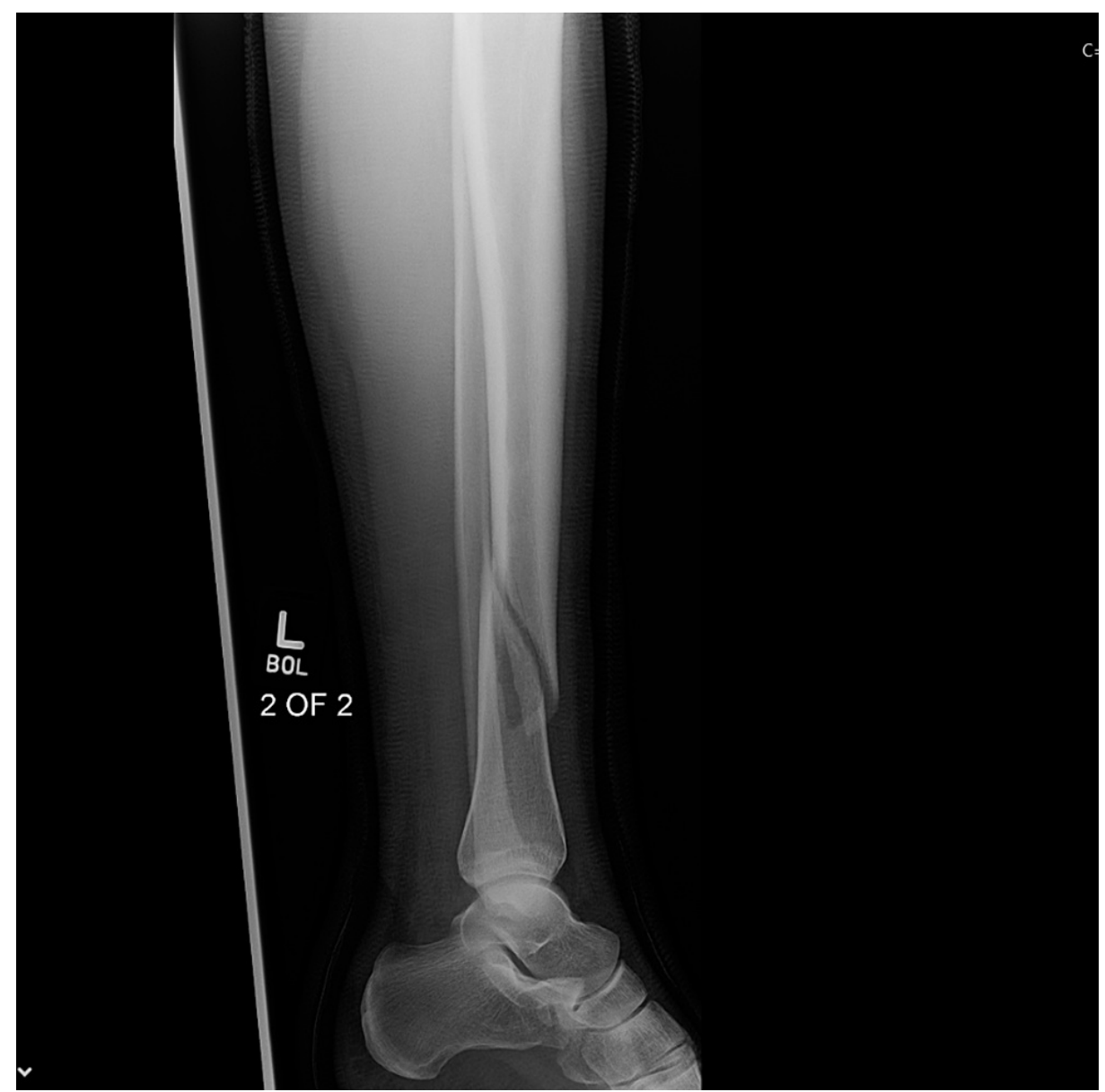

FIGURE 4: Distal Tibia fracture at the time of initiation of LIPUS therapy (6 months post injury - lateral view)

LIPUS - Low-Intensity Pulsed Ultrasound 


\section{Cureus}

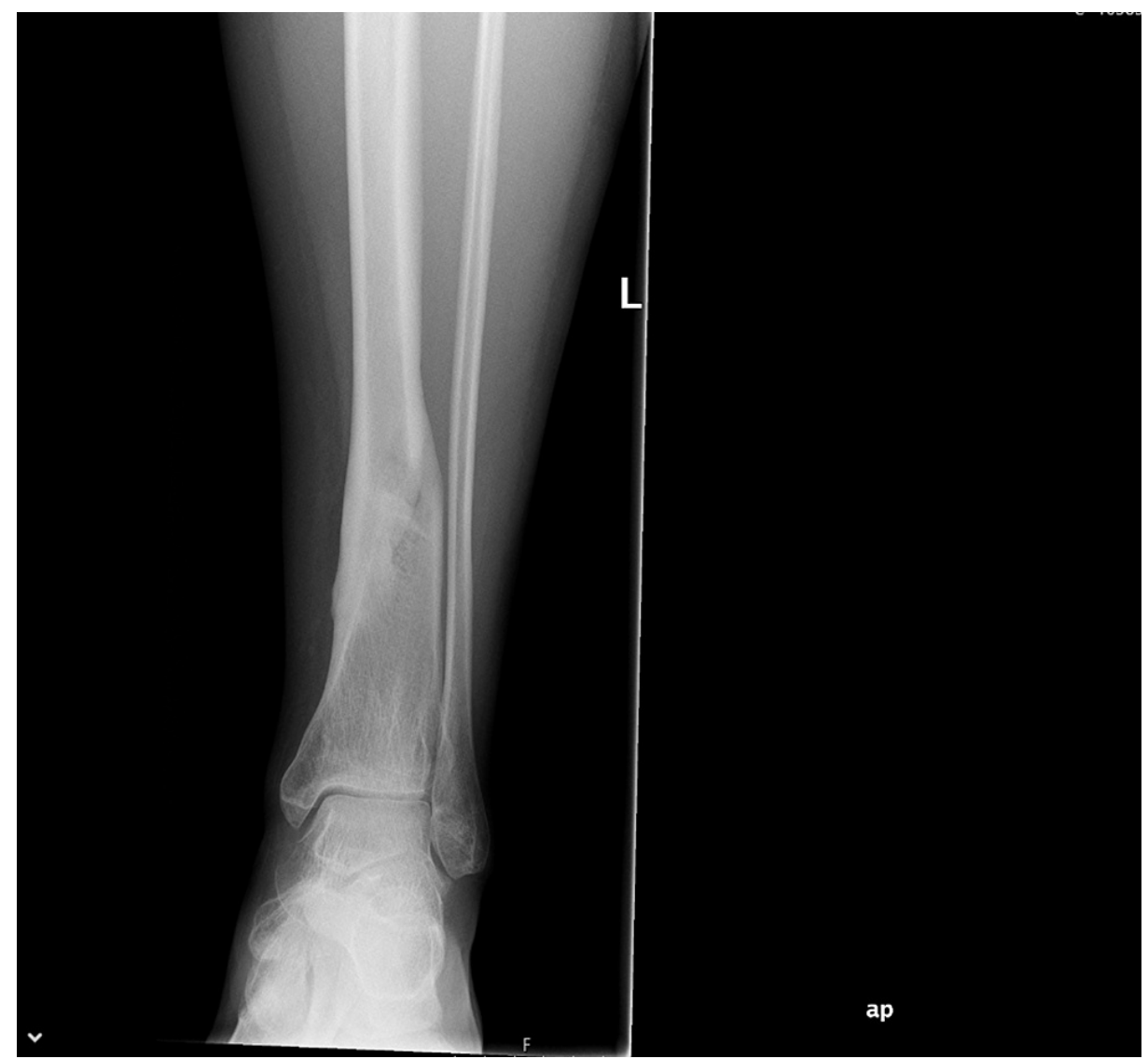

FIGURE 5: Union status at the completion of 6 months of LIPUS therapy (AP View)

AP - Anteroposterior; LIPUS - Low-Intensity Pulsed Ultrasound 


\section{Cureus}

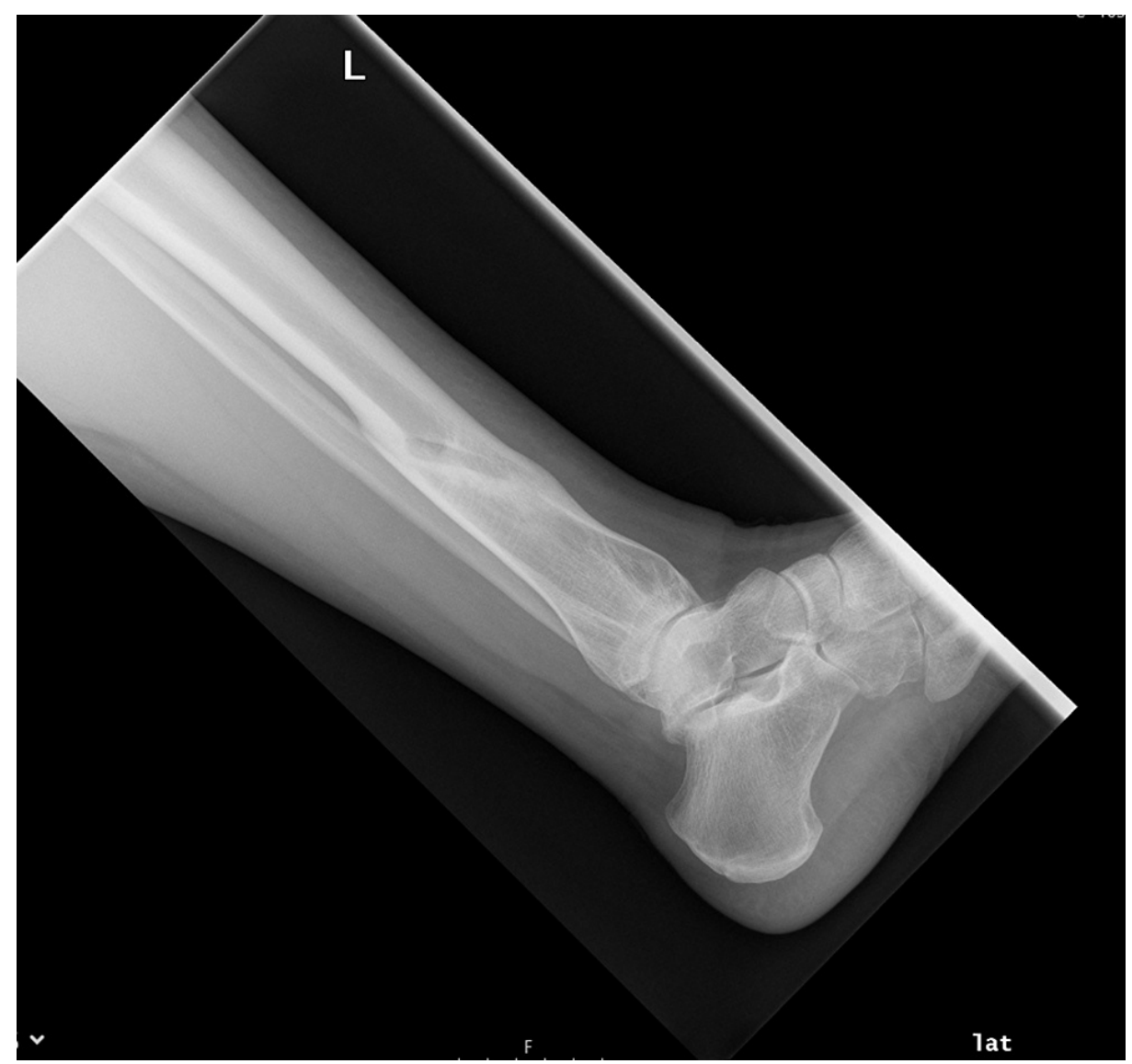

FIGURE 6: Union status at the completion of 6 months of LIPUS therapy (Lateral view)

LIPUS - Low-Intensity Pulsed Ultrasound

The pre-LIPUS therapy mean VAS score was found to be 5.1 (range 3-7). This improved to 3.9 (range 0-6) upon culmination of LIPUS therapy in the 42 compliant patients. The nonunions associated with fractures that failed to unite comprised both atrophic and hypertrophic nonunion. The nonunion post Proximal Tibia ORIF, midshaft Femur ORIF, and Distal Femur ORIF was of the hypertrophic type, while the remaining were atrophic type. The maximum fracture site gap in the non-united fractures was found to be not less than $9 \mathrm{~mm}$ (range $9-10 \mathrm{~mm}$ ) at the commencement of LIPUS therapy. This may suggest that a fracture site gap less than $9 \mathrm{~mm}$ has a positive role in predicting the success of LIPUS therapy.

\section{Discussion}

Low-intensity pulsed ultrasound has been drawing increased attention globally owing to its therapeutic benefits while avoiding any significant risks like surface temperature change caused by the use of highintensity ultrasound in humans [14]. National Institute for Health and Care Excellence (NICE) in the United Kingdom (UK) has also supported the use of LIPUS therapy in nonunions and delayed unions under controlled settings while suggesting further research to study its mechanism of action, principle, and efficacy [15]. A multi-centre randomised controlled trial (RCT) conducted in Germany reported better healing in patients who had LIPUS therapy by a margin of $34 \%$ as compared to the control or sham group [16]. Given the fact that established nonunions show no signs of spontaneous healing, the findings of LIPUS therapy in various studies around the world are compelling. Bashardoust et al. in a meta-analysis reported that patients who received LIPUS therapy exhibited accelerated radiographic union in 14 out of the 20 studies reviewed [17]. Bawale et al. have also compared success rates of LIPUS therapy to success rates of surgical management of nonunions, with both having reportedly similar success rate of around 70\% [4]. This also opens up the debate of whether attempting surgical management of an established nonunion is a better option than trying LIPUS therapy provided both have similar success rates and LIPUS therapy may comparatively be much safer and sometimes even faster. Jingushi et al. suggested a significant relationship between union rate and the period from the most recent operation [18]. They have suggested that LIPUS therapy be initiated within six months of the most recent operation in all cases of post-operative nonunion and delayed union owing to the high efficacy, safety, and tolerability of the therapy. The cost of LIPUS therapy is also estimated to be almost $49 \%$ less than the cost of surgical revision in the National Health 
Services (NHS) of the United Kingdom [15]. Thus, LIPUS therapy can also be projected as an economic and viable alternative to revision surgery provided it is proposed to have similar success rates as revision surgery. It has been projected that LIPUS therapy can save almost $£ 2200$ per patient who does not undergo revision surgery. In a resource stretched and heavily burdened NHS, this may well prove to be a very costeffective alternative to surgeries.

Although it has been widely published that LIPUS therapy may be effective in reducing time to radiographic union, its role in actually providing a beneficial or positive effect through accelerated functional recovery is still unclear. Promoting radiographic union may not directly correlate to improved functional levels or clinical healing. Hence, although the role of LIPUS in promoting radiographic union may be explained to some extent, the role of LIPUS in the clinical healing of nonunions and delayed unions is still debatable and not well understood [12]. Jiang et al. in their systematic review found that LIPUS therapy had a positive effect on chronic delayed unions and nonunions which had not healed for almost 10 years, but were still unable to comment on the role of LIPUS therapy in clinical healing [14]. Thus, the role of LIPUS therapy in clinical healing needs to be examined further.

\section{Conclusions}

Our series demonstrates a success rate of $78.57 \%$ with the use of LIPUS therapy in the treatment of nonunion or delayed union. This is in line with the success rates that have been reported in the current literature. The LIPUS therapy union rate is also comparable to the union rates obtained following surgical revisions of nonunions and delayed unions. As outcomes are comparable, one may consider LIPUS therapy over surgical treatment since safety favours LIPUS therapy owing to its non-invasiveness. However, large multi-centre studies may be needed to confirm our findings before large scale adoption of LIPUS therapy can be recommended. The main limitation of our study is that it is a single-centre clinical series. Multi-centre studies and randomised controlled trials may be needed to study the efficacy and indications of LIPUS therapy in detail. Although, current evidence supports the use of LIPUS therapy to promote radiographic union of delayed unions and nonunions, further studies are required to delineate the exact role of LIPUS therapy in clinical healing along with further studies to understand the mechanism of action of LIPUS therapy in nonunions and delayed unions.

\section{Additional Information \\ Disclosures}

Human subjects: Consent was obtained or waived by all participants in this study. Surgical Directorate IRB, Barts Health NHS Trust issued approval STUDY/1/KB/1006. "At the Institutional Ethics Review Meeting held on January 4, 2016, your research proposal was examined and discussed. After due consideration, the board has decided to approve your above-mentioned protocol to be conducted as per the protocol submitted". Animal subjects: All authors have confirmed that this study did not involve animal subjects or tissue. Conflicts of interest: In compliance with the ICMJE uniform disclosure form, all authors declare the following: Payment/services info: All authors have declared that no financial support was received from any organization for the submitted work. Financial relationships: All authors have declared that they have no financial relationships at present or within the previous three years with any organizations that might have an interest in the submitted work. Other relationships: All authors have declared that there are no other relationships or activities that could appear to have influenced the submitted work.

\section{References}

1. Zura R, Xiong Z, Einhorn T, et al.: Epidemiology of fracture nonunion in 18 human bones . JAMA Surg. 2016, 151:e162775. 10.1001/jamasurg.2016.2775

2. Bishop JA, Palanca AA, Bellino MJ, Lowenberg DW: Assessment of compromised fracture healing. J Am Acad Orthop Surg. 2012, 20:273-82. 10.5435/JAAOS-20-05-273

3. McKinley TO, Gaski GE, Vodovotz Y, Corona BT, Billiar TR: Diagnosis and management of polytraumatized patients with severe extremity trauma. J Orthop Trauma. 2018, 32 Suppl 1:S1-6. 10.1097/BOT.0000000000001114

4. Bawale R, Segmeister M, Sinha S, Shariff S, Singh B: Experience of an isolated use of low-intensity pulsed ultrasound therapy on fracture healing in established non-unions: a prospective case series. J Ultrasound. 2020, 10.1007/s40477-020-00464-9

5. Tian R, Zheng F, Zhao W, Zhang Y, Yuan J, Zhang B, Li L: Prevalence and influencing factors of nonunion in patients with tibial fracture: systematic review and meta-analysis. J Orthop Surg Res. 2020, 15:377. 10.1186/s13018-020-01904-2

6. Nicholson JA, Tsang ST, MacGillivray TJ, Perks F, Simpson AH: What is the role of ultrasound in fracture management?: Diagnosis and therapeutic potential for fractures, delayed unions, and fracture-related infection. Bone Joint Res. 2019, 8:304-12. 10.1302/2046-3758.87.BJR-2018-0215.R2

7. Wood RW, Loomis AL: The physical and biological effects of high-frequency soundwaves of great intensity . Lond Edinb Dublin Philos Mag J Sci. 1927, 4:417-36. 10.1080/14786440908564348

8. Ricardo M: The effect of ultrasound on the healing of muscle-pediculated bone graft in scaphoid non-union . Int Orthop. 2006, 30:123-7. 10.1007/s00264-005-0034-2

9. Harrison A, Lin S, Pounder N, Mikuni-Takagaki Y: Mode \& mechanism of low intensity pulsed ultrasound (LIPUS) in fracture repair. Ultrasonics. 2016, 70:45-52. 10.1016/j.ultras.2016.03.016 


\section{Cureus}

10. Romano CL, Romano D, Logoluso N: Low-intensity pulsed ultrasound for the treatment of bone delayed union or nonunion: a review. Ultrasound Med Biol. 2009, 35:529-36. 10.1016/j.ultrasmedbio.2008.09.029

11. Leighton R, Watson JT, Giannoudis P, Papakostidis C, Harrison A, Steen RG: Healing of fracture nonunions treated with low-intensity pulsed ultrasound (LIPUS): a systematic review and meta-analysis. Injury. 2017, 48:1339-47. 10.1016/j.injury.2017.05.016

12. Rutten S, van den Bekerom MP, Sierevelt IN, Nolte PA: Enhancement of Bone-Healing by Low-Intensity Pulsed Ultrasound: A Systematic Review. JBJS Rev. 2016, 4:10.2106/JBJS.RVW.0.00027

13. Berber R, Aziz S, Simkins J, Lin SS, Mangwani J: Low intensity pulsed ultrasound therapy (LIPUS): a review of evidence and potential applications in diabetics. J Clin Orthop Trauma. 2020, 11:S500-5. 10.1016/j.jcot.2020.03.009

14. Jiang X, Savchenko O, Li Y, Qi S, Yang T, Zhang W, Chen J: A review of low-intensity pulsed ultrasound for therapeutic applications. IEEE Trans Biomed Eng. 2019, 66:2704-18. 10.1109/TBME.2018.2889669

15. Majeed H, Karim T, Davenport J, Karski M, Smith R, Clough TM: Clinical and patient-reported outcomes following low intensity pulsed ultrasound (LIPUS, Exogen) for established post-traumatic and post-surgical nonunion in the foot and ankle. Foot Ankle Surg. 2020, 26:405-11. 10.1016/j.fas.2019.05.009

16. Schofer MD, Block JE, Aigner J, Schmelz A: Improved healing response in delayed unions of the tibia with low-intensity pulsed ultrasound: results of a randomized sham-controlled trial. BMC Musculoskelet Disord. 2010, 11:229. 10.1186/1471-2474-11-229

17. Bashardoust Tajali S, Houghton P, MacDermid JC, Grewal R: Effects of low-intensity pulsed ultrasound therapy on fracture healing: a systematic review and meta-analysis. Am J Phys Med Rehabil. 2012, 91:34967. 10.1097/PHM.0b013e31822419ba

18. Jingushi S, Mizuno K, Matsushita T, Itoman M: Low-intensity pulsed ultrasound treatment for postoperative delayed union or nonunion of long bone fractures. J Orthop Sci. 2007, 12:35-41. 10.1007/s00776-006-1080-3 